\title{
Utility of Dual-Energy CT in Abdominal Interventions
}

\author{
Tara Prasad Tripathy ${ }^{1}$ Ranjan Patel ${ }^{1}{ }^{10} \quad$ Karamvir Chandel $^{1} \quad$ Amar Mukund $^{1}$ \\ ${ }^{1}$ Department of Interventional Radiology, Institute of Liver \& Biliary \\ Sciences, New Delhi, India \\ J Gastrointestinal Abdominal Radiol ISGAR 2022;5:127-134. \\ Address for correspondence Amar Mukund, MD, Department of \\ Interventional Radiology, Institute of Liver \& Biliary Sciences, D-1 \\ Vasant Kunj, New Delhi 110070, India \\ (e-mail: dramarmukund@gmail.com).
}

\begin{abstract}
Keywords

- dual-energy CT

- abdominal interventions

- transarterial chemoembolization

- transjugular intrahepatic portosystemic shunt

- CT-guided interventions

Dual-energy computed tomography (DECT) is an emerging CT technique based on data acquisition at two different settings. Various postprocessing techniques generate different sets of images, each with unique advantages. With DECT, it is possible to obtain virtual unenhanced images from monochromatic reconstructions and attenuation maps of different elements, thereby improving the detection and characterization of a variety of lesions. Presently, DECT is widely used to evaluate pulmonary embolism, characterize abdominal masses, determine the composition of urinary calculi, and detect tophi in gout. CT angiography is an essential prerequisite for endovascular intervention. DECT allows a better quality of angiographic images with a lesser dose of contrast. Various postprocessing techniques in DECT also help in a better evaluation of response to locoregional therapy. Virtual noncontrast images and iodine map differentiate residual or recurrent tumors from intrinsically hyperdense materials. Superior metallic artifact reduction allows better evaluation of vascular injuries adjacent to bony fractured fragments or previously deployed embolization coils. In addition to metal artifacts reduction, virtual monochromatic spectral imaging could further mitigate metal artifacts during CT-guided biopsy, providing an improved depiction of lesions and safe and versatile access for long puncture pathways. This article reviews and illustrates the different applications of DECT in various abdominal interventions. Familiarity with the capabilities of DECT may help interventional radiologists to improve their practice and ameliorate patient care.
\end{abstract}

\section{Introduction}

Dual-energy computed tomography (DECT) is an innovative imaging technique based on data acquisition at two different energy settings. The attenuation of X-ray photons differs at different energy settings. Based on the attenuation differences at different energies, it is possible to differentiate materials of different effective atomic numbers. ${ }^{1,2}$ The capa- bility of obtaining different material-specific data sets, that is, virtual noncontrast (VNC) image, iodine map, and monochromatic images, in a single acquisition improves detection and characterization of different pathologies. ${ }^{3}$ Furthermore, fast and superior processing of angiographic images by utilizing material-specific subtraction in DECT enables better depiction of vascular anatomy and pathologies. ${ }^{4}$ DECT also offers superior metallic artifact reduction and thus improves published online January 24, 2022
DOI https://doi.org/ 10.1055/s-0041-1740475. ISSN 2581-9933.

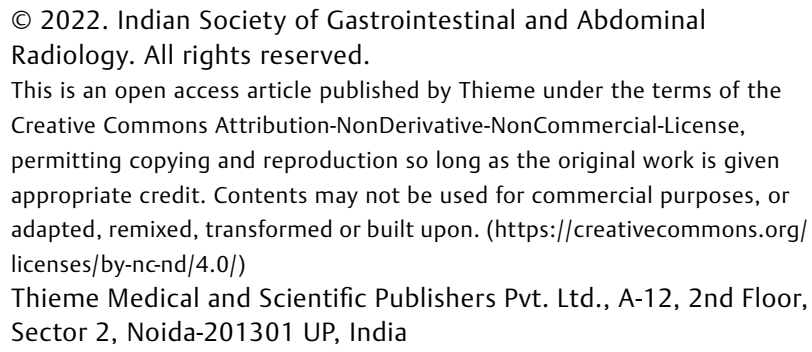

(C) 2022. Indian Society of Gastrointestinal and Abdominal Radiology. All rights reserved.

This is an open access article published by Thieme under the terms of the Creative Commons Attribution-NonDerivative-NonCommercial-License, permitting copying and reproduction so long as the original work is given appropriate credit. Contents may not be used for commercial purposes, or adapted, remixed, transformed or built upon. (https://creativecommons.org/ licenses/by-nc-nd/4.0/)

Thieme Medical and Scientific Publishers Pvt. Ltd., A-12, 2nd Floor, Sector 2, Noida-201301 UP, India 
in image quality of soft tissue adjacent to metallic implants or devices (e.g., pelvic structure adjacent to metallic hip prosthesis). ${ }^{5}$ In clinical practice, DECT is widely used in cardiovascular imaging, especially in evaluating pulmonary thromboembolism and myocardial ischaemia. ${ }^{6}$ Other common uses of DECT include the identification of tophi in gouty arthritis and determining renal calculi composition. ${ }^{5}$ Studies have demonstrated improvement in detecting and characterization of various renal, adrenal, hepatic, and pancreatic abnormalities. $^{3}$ In addition, current dual-energy scanners offer an improved temporal resolution, which is very helpful for CT angiography (CTA), and increased photon flux that improves image quality in large or obese patients. Presently, the use of DECT has also been extended to various interventional procedures. Different postacquisition image processing techniques guide an interventional radiologist for decision making and aid in evaluating treatment response. This article reviews and illustrates the different applications of DECT in various abdominal interventions.

\section{General Principle of DECT}

The attenuation of X-rays depends on the composition of the matter and energy of the incident X-ray photons. ${ }^{7}$ DECT is primarily based on the concept of photoelectric effect. Photoelectric effect increases with increasing atomic number. Due to increased photoelectric absorption, X-ray photons exhibit a spike in attenuation at K-edge, that is, at energy levels just greater than the k-shell binding energy of the interacting matter. Iodine has K-edge value closer to the mean energy of the X-ray spectrum, thus causes maximum attenuation. This property makes it not only an effective CT contrast agent but also a prime target for DECT applications. DECT is based on the simultaneous acquisition at low (80 $\mathrm{kVp}$ ) and high (140 kVp) energy levels. ${ }^{7} \mathrm{DECT}$ is capable of differentiating materials based on their different energydependent X-ray absorption. Several different techniques are utilized to achieve simultaneous data acquisition at both energies, including dual-source DECT (DS-DECT), singlesource DECT (SS-DECT), and single-source dual-energy scanner with dual detector layers. ${ }^{8}$

\section{DECT Postprocessing Techniques}

Most commonly, "conventional" images are generated through an appropriate combination of low- and high-energy data set. These images closely simulate that of singleenergy CT (SECT). In addition, postacquisition data processing yields several types of images, each with unique advantages.

1. VNC images: It obviates the need for an additional noncontrast CT (NCCT) image acquisition, thereby reducing radiation exposure. ${ }^{9}$

2. Iodine map: It shows the iodine-containing area as bright, whereas unenhanced areas remain dark, thus differentiating true enhancement from pseudoenhancement. ${ }^{10}$

3. Virtual monochromatic imaging: Monochromatic images represent reconstructed images obtained at different fixed voltages, ranging from 40 to $140 \mathrm{keV}$. Virtual monochromatic images at lower $\mathrm{kVp}$ show higher image contrast, making small enhancing lesions more conspicuous but with more image noise. ${ }^{11}$

4. Metal artifacts reduction: DECT reduces the metallic artifacts (due to beam hardening) and provides satisfactory visualization of metallic implants and surrounding bony structures on higher $\mathrm{kVp}$ monochromatic images. This is achieved by using metal artifact reduction software. ${ }^{12} \mathrm{SS}$ DECT with fast kilovoltage switching enables superior beam hardening correction than that of the DECT system.

5. Attenuation maps of different elements: Dual-energy acquisition makes it possible to distinguish elements such as iodine, calcium, water, and uric acid based on their atomic number. $^{13}$

\section{Radiation Dose Considerations}

In reality, overall radiation dose in DECT is equivalent to or often lower than that of conventional imaging. This is achieved by splitting the overall radiation dose into the high- and low-energy components in such a way that result in reduction of total radiation dose. Furthermore, DECT can be used to eliminate additional scan phases in traditionally multiphase examinations. It can also eliminate the radiation exposure of additional scans that might otherwise be needed to characterize lesions. A study that included patients with suspected hepatocellular carcinoma showed a highly significant reduction in dose-length product and $37 \%$ reduction in effective dose $(p<0.001)$ for an adapted dual-energy acquisition protocol compared with single-energy acquisition. ${ }^{14}$ Virtual unenhanced image acquisition reduces the radiation dose up to $47 \%$, depending on the protocol, while obtaining images of equivalent quality. ${ }^{9}$

\section{Applications of DECT in Abdominal Interventions}

Various postacquisition processing of dual-energy data sets improves lesion detection and characterization as well as helps in the posttreatment response evaluation. High-quality angiographic images with simultaneous reduction of metallic artifacts provide a more accurate diagnosis of various vascular pathologies and guide the interventional radiologist in treatment planning. DECT also aids in follow-up after interventional procedures.

\section{Preintervention Vascular Roadmap and Delineation of Vascular Pathologies}

Dual-energy applications of aortic imaging can also be applied to CTA of visceral arteries, such as the hepatic, splenic, and renal arteries. Iodine-selective or bone-masked angiographic visualization of the vasculature, supplemented by low $\mathrm{kVp}$ imaging, provides adequate anatomical delineation in instances of poor vascular opacification and smaller arteries (-Fig. 1). Furthermore, an iodine map can differentiate a vascular pseudoaneurysm from an adjacent hyperdense hematoma. Pseudoaneurysm/aneurysm shows uptake of color on the iodine map, where the adjacent hematoma does not ${ }^{15}$ (-Fig. 2D and G). 


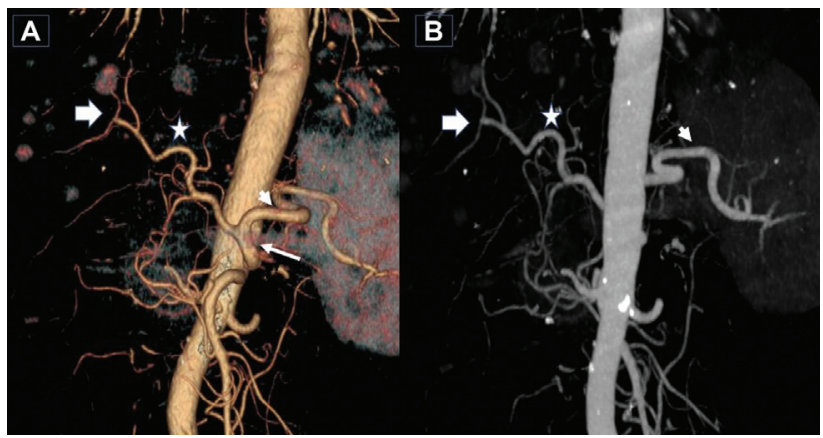

Fig. 1 Dual-source dual-energy bone-subtracted volume-rendered angiography $(A)$ and maximum intensity projection image $(B)$ of abdominal aorta and its branches. Celiac axis and its branching pattern with vascular roadmap may be used for pre-transarterial chemoembolization (TACE) assessment. Bone subtraction imaging and subsequent three-dimensional (3D) rendering enables rapid and effective visual display of aorta and its branches without bone overlay (arrow: celiac artery; arrow head: splenic artery; asterisk: right hepatic artery; block arrow: branch of right hepatic artery supplying hepatocellular carcinoma [HCC]).

Beam hardening and blooming artifacts from the atherosclerotic calcification often limits the accurate assessment of degree of arterial stenosis on conventional single-energy CTA. Severe vascular calcification usually overestimates the arterial stenosis. ${ }^{16}$ DECT offers a precise assessment of luminal and ostial stenosis in atherosclerotic celiac artery, superior mesenteric artery (SMA), and renal artery. This is important before planning for angioplasty and/or stenting. Owing to the material-specific subtraction capability of the
DECT, calcification can be subtracted on iodine/calcium or iodine/hydroxyapatite material density images to improve luminal delineation on angiographic images. ${ }^{17}$ Additionally, dual-source DECT can provide automated, faster, and more accurate bone subtraction compared with threshold-based bone subtraction. This further improves the assessment of the vascular lumen. ${ }^{18}$

\section{Hepatic Intervention}

VNC images are of acceptable image quality and show a good correlation with that of conventional CT images. Additional NCCT is thus not required during multiphasic CT for hepatic lesion characterization and avoids additional radiation exposure. ${ }^{19}$ At times, smaller hepatic focal lesions may not be identified on conventional CECT. Low kVp monoenergetic images increase the lesion conspicuity due to high contrast and seem very useful in detecting these smaller lesions ${ }^{11}$ (-Fig. 3). Using DECT, a small hepatocellular carcinoma can be differentiated from a small hemangioma with a high degree of sensitivity and specificity by quantifying the iodine density of lesions, first during the arterial and then during the portal venous phase. ${ }^{20,21}$ Proper lesion characterization is essential before targeting it for biopsy and locoregional therapy, such as microwave ablation (MWA) and transarterial chemoembolization (TACE).

The high attenuation of deposited lipiodol can mask residual or recurrent tumor after TACE. An NCCT might be helpful to assess the viability around the lipiodol uptake. VNC could be a useful measure in such situation without additional radiation dose. Color-coded iodine CT (CICT) is seen to

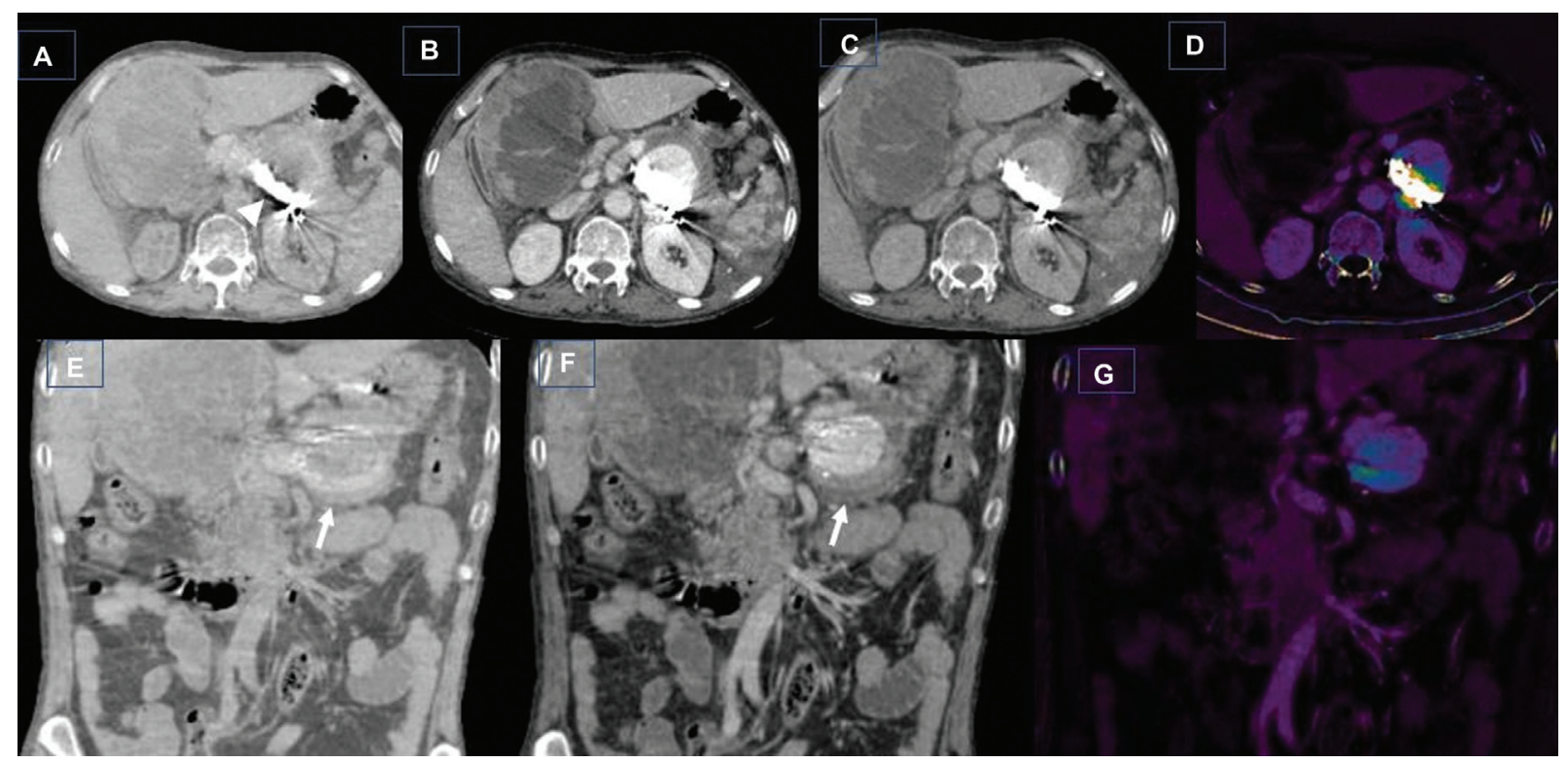

Fig. 2 Postembolization dual-energy computed tomography (DECT) abdomen of a patient of liver cirrhosis, portal hypertension, and a large hepatocellular carcinoma in segment IV. Patient underwent embolization of a large splenic artery aneurysm ( $>2 \mathrm{~cm})$ using coils and glue. (A) Virtual unenhanced (VNC) image shows coil and glue cast (arrowhead) with mildly hyperdense aneurysmal sac. (B) Monochromatic arterial phase image at $65 \mathrm{keV}$ shows more conspicuous enhancement of residual aneurysm. (C) Monochromatic arterial phase image at $100 \mathrm{keV}$ shows reduction in coils and glue cast-related artifacts compared with $65 \mathrm{keV}$ image. (D) lodine map of corresponding axial section. (E) Virtual unenhanced image coronal reconstruction showing eccentric hyperdense thrombus (arrow). (F, G) Coronal arterial phase and iodine map. Virtual unenhanced reconstruction images are similar to unenhanced images. The visualization of the aneurysm sac is good with reconstructions at a low level of energy (on B) and iodine mapping (on D). At a high-energy setting the aneurysm sac is less conspicuous due to the sharp reduction of contrast-to-noise ratio. 


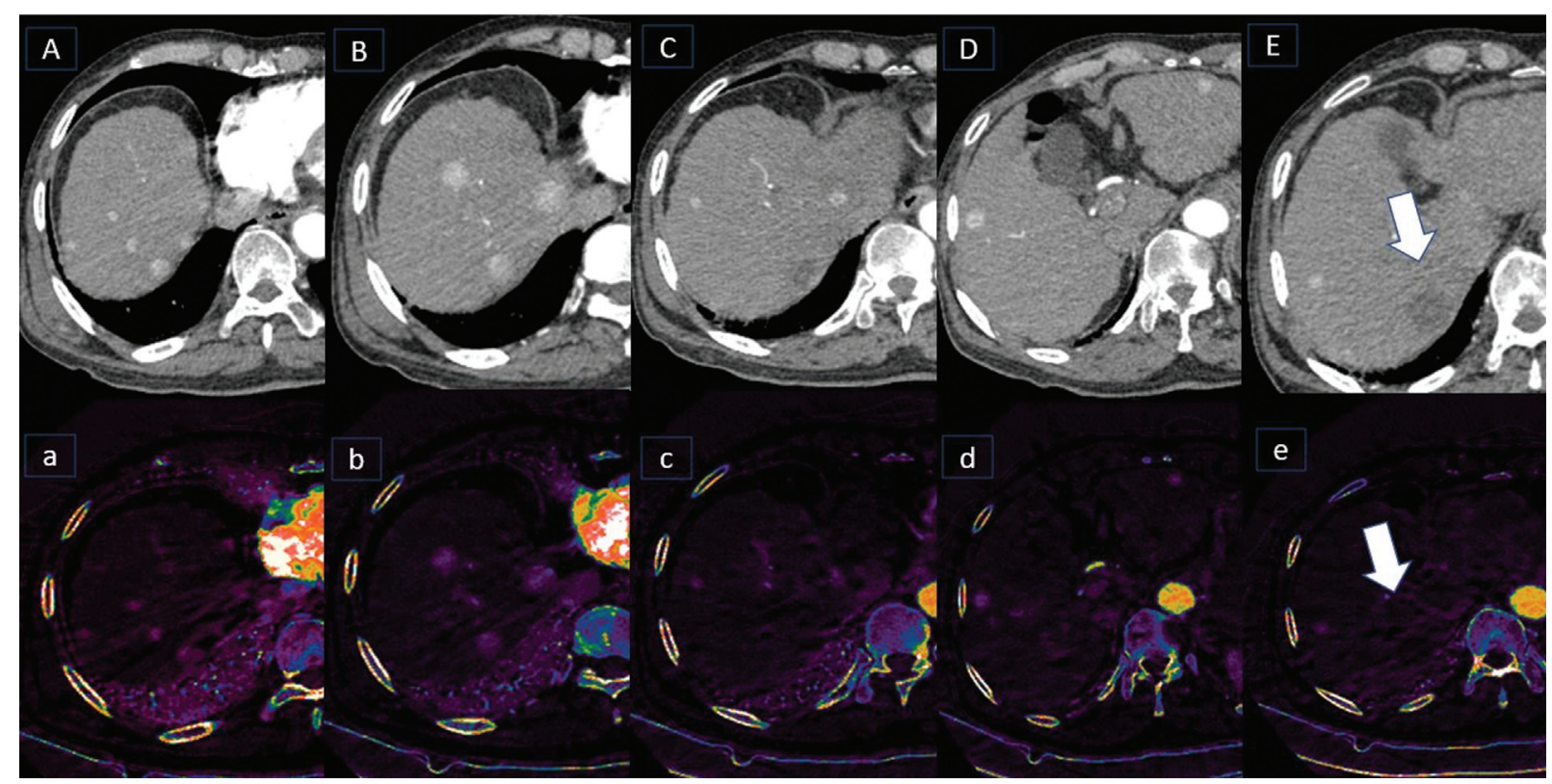

Fig. 3 Follow-up dual-energy computed tomography (DECT) in a 56-year-old man who was treated with drug-eluting bead transarterial chemoembolization (TACE) for segment VII hepatocellular carcinoma (HCC) (E, e). Contrast-enhanced CT images obtained in the arterial phase at lower energy levels, $65 \mathrm{keV}$ (top row, A-E) shows several (at least 12) enhancing liver nodules. Corresponding iodine images (with a color overlay; bottom row, a-e) show higher lesion-to-parenchyma contrast with improved lesion conspicuity (arrow, A, a), providing better depiction of nodules with varying enhancement patterns in a cirrhotic liver. Thus, patient had progressive disease with multifocal HCCs without local tumor progression.

be helpful in more accurate assessment of viable tumor components in follow-up imaging after conventional TACE. Lee et al described a CICT by limiting the window of iodine map from the Hounsfield unit of liver parenchyma to that of the aorta, thus excluding the strong attenuation of compact lipiodol as a black area. At the same time, viable hypervascular tumor components were color-coded, improving viable tumor to lipiodol contrast ${ }^{22}$ (-Fig. 4).

Iso- or hyperdense necrotic or hemorrhagic content in the treated lesion (radiofrequency ablation/MWA, drug-eluting bead TACE) simulate viable residual tumor on follow-up CT. In such a situation, VNC and iodine map is very helpful in differentiating the viable tumor component from the nonviable part. The viable tumor shows uptake in iodine map and increases in attenuation on contrast-enhanced image compared with VNC (-Fig. 5).

\section{Gastrointestinal Bleed}

In suspected gastrointestinal bleeding, differentiation of high-attenuation hematoma or intraluminal bowel content from actively extravasating iodine can be extremely helpful. VNC rules out the presence of luminal hyperdense materials, and simultaneously iodine map shows the active contrast extravasation as a colored area. These postprocessing techniques eliminate the need for additional NCCT and delayed phase imaging to show progressive enhancement. ${ }^{23-25}$ The source of rebleeding is often inaccurately depicted on conventional CT due to metallic beam hardening artifacts from the previously deployed embolizing materials or surgical clips. DECT is found to be very useful in identifying the site of bleeding and feeder arteries, utilizing the benefits of metallic artifacts reduction algorithm and iodine map (-Fig. 2). Subtraction of monochromatic images between two energy levels can subtract coils composed of dense metallic materials and highlights the pathology.

\section{Abdominal and Pelvic Trauma}

Active bleeding due to vascular injuries in trauma patients requires urgent intervention, preferably through the endovascular approach. Detection of vascular anatomy, site of vascular injury, size, and morphology of pseudoaneurysm on CT are essential prerequisites before planning for endovascular embolization. Active contrast extravasation typically appears as a jet of contrast having attenuation similar to that of adjacent vasculature with a change in the morphology in delayed phase imaging. However, varying appearances may be seen on imaging. It is difficult to differentiate whether high attenuation material is due to active extravasation, pseudoaneurysm, or other hyperdense materials, such as bone fragments. A worth mentioning clinical situation is active bleeding in the vicinity of a pelvic fracture, where hyperdense bony fragments may obscure bleeding. ${ }^{26}$ Analysis of VNC and iodine map helps distinguish active extravasation from bony fragments. Bony fragments are seen in both VNC and iodine map, but extravasation is only seen in the iodine map. ${ }^{27}$ Low $\mathrm{kVp}$ monochromatic images also increase the conspicuity of vascular injury.

\section{Evaluation of Abdominal Aortic Endoleaks}

Endoleaks are a common complication after endovascular aneurysm repair that may further enlarge the aneurysm, and 


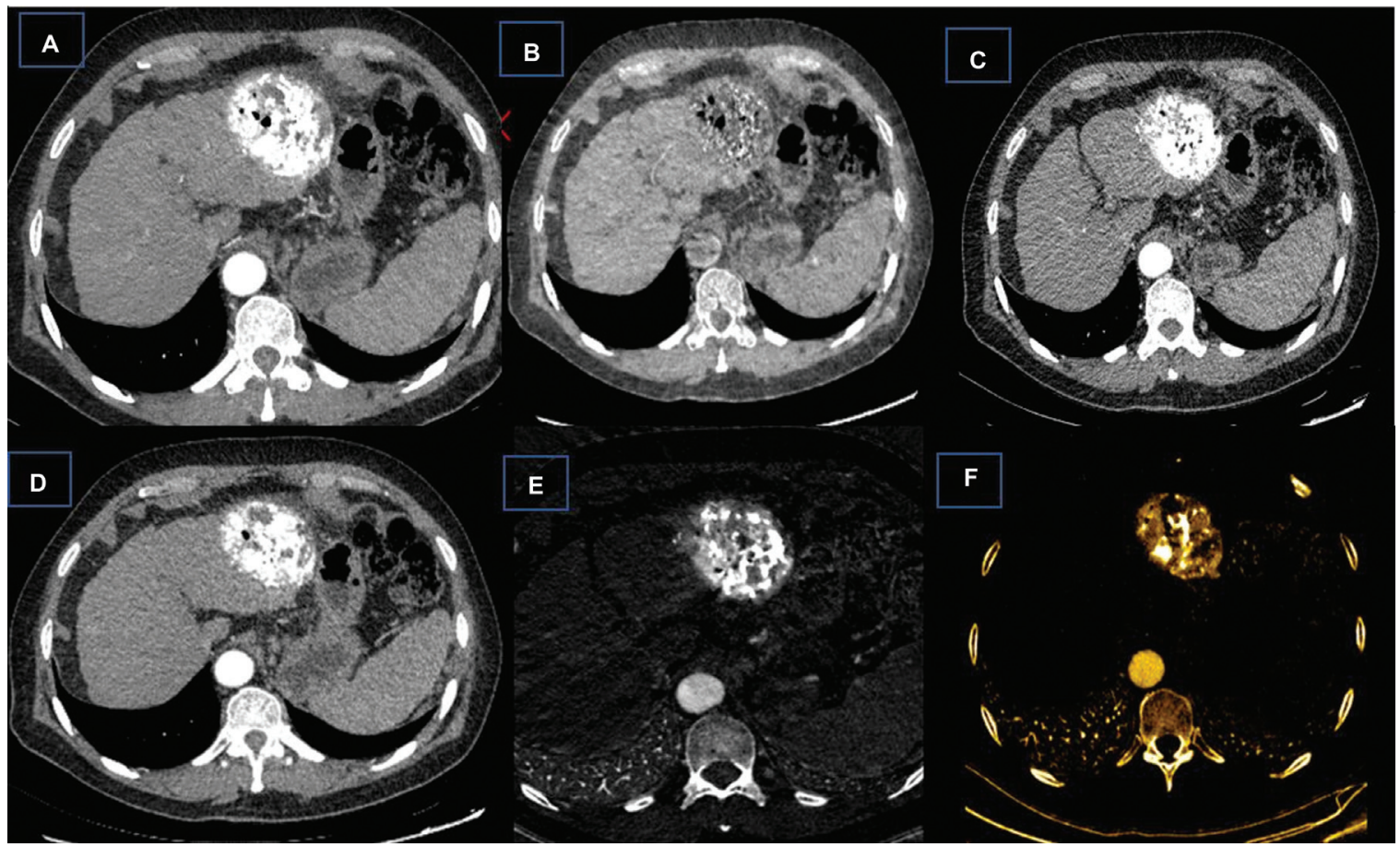

Fig. 4 Triple phase contrast-enhanced dual-energy computed tomography (DECT) of the abdomen performed for a 59-year-old patient who has been treated with conventional transarterial chemoembolization (TACE) for left lobe hepatocellular carcinoma (HCC). Lipiodol deposition seen within the lesion. (A) Native polychromatic image (arterial phase). (B) Virtual unenhanced image. (C) Monochromatic reconstruction at 65 keV (arterial phase). (D) Monochromatic reconstruction at $100 \mathrm{keV}$ (arterial phase) (E) lodine map of arterial phase. (F) Color overlay of iodine map. Virtual unenhanced reconstruction images are similar to unenhanced images. The arterial enhancement was inconspicuous in the background of lipiodol. The enhancement was convincingly seen in the arterial phase iodine maps suggestive of recurrence of HCC.

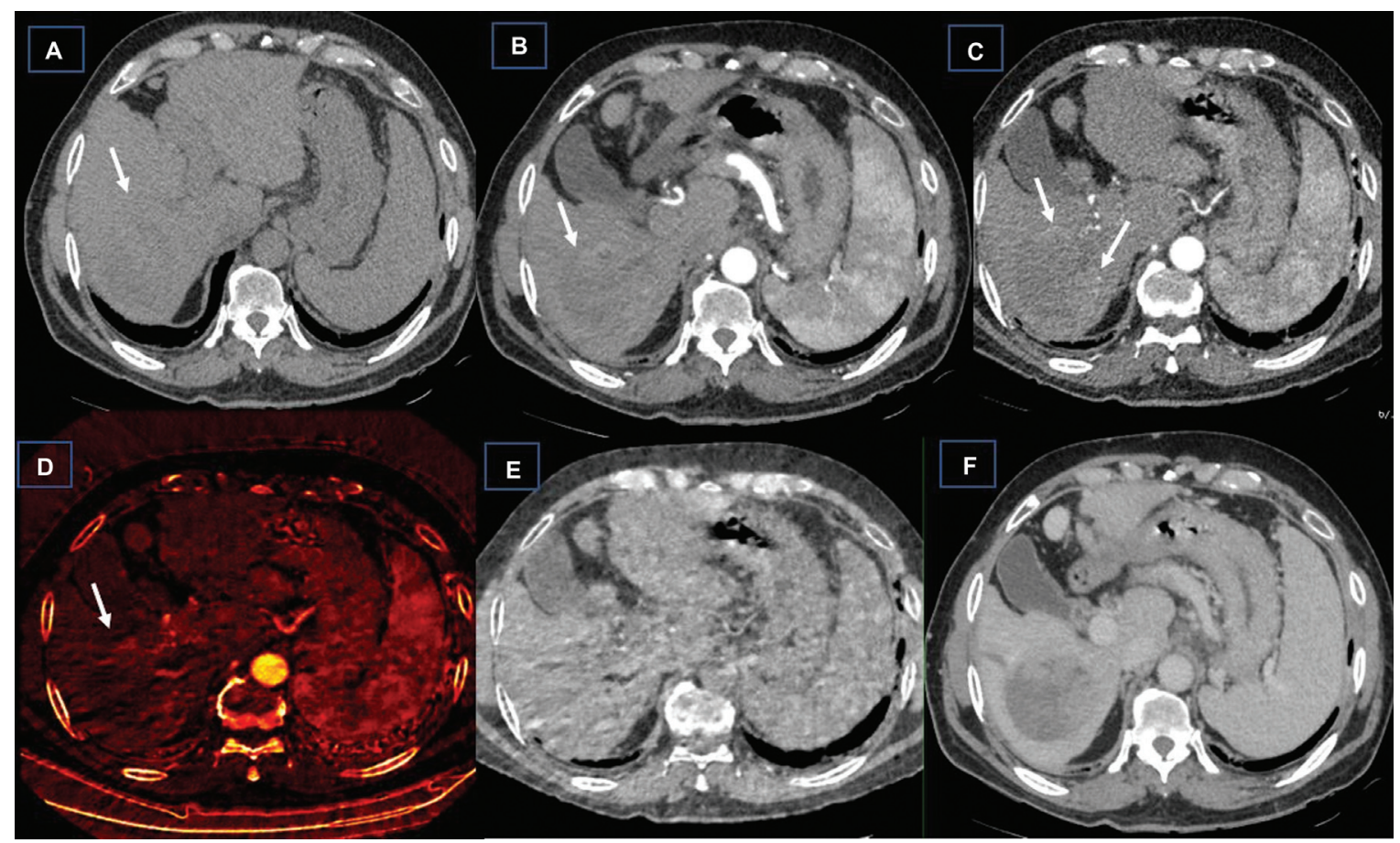

Fig. 5 Dual-energy computed tomography (DECT) of the abdomen performed for the follow-up of microwave ablation for hepatocellular carcinoma (HCC) in segment $\mathrm{VI}$ in a 60-year-old man with progressive increase in serum alpha-fetoprotein (AFP) levels. (A) Noncontrast scan showing subtle hyperdense areas in the periphery of ablated lesion. (B) Native polychromatic arterial phase image. (C) Monochromatic reconstruction at $65 \mathrm{keV}$; showing subtle peripheral nodular enhancement (D) lodine map with color overlay confirming iodine uptake in periphery of the lesion. (E) Virtual noncontrast image at same level. (F) Venous phase: peripheral areas of the ablated lesion show hyperdensities which may be hemorrhagic component or postablation changes. The visualization of the recurrence in lesion periphery is good with reconstructions at a lower level of energy (on C) and iodine mapping (on D). 
thus, increase the risk of rupture. Early detection is important to avoid fatal consequences. Although the optimal protocol for aortic evaluation after endovascular stent repair is not well-established, biphasic or triphasic protocols (unenhanced, arterial, and delayed) are usually advocated to detect and characterize endoleaks adequately. ${ }^{28-31}$ Interestingly, few recent studies have shown the advantages of VNC and low-energy monochromatic images in the detection of endoleaks with low radiation exposure. Chandarana et al reported the detection of all endoleaks on delayed venous phase DECT imaging alone, utilizing the benefits of VNC and $80 \mathrm{kVp}$ images. ${ }^{32} \mathrm{~A}$ mean dose reduction of 27.8 to $11.1 \mathrm{mSv}$ was achieved by eliminating the unenhanced and arterial phases. Similarly, Stolzmann et al illustrated that a single delayed DECT acquisition was sufficient, with very high sensitivity, specificity, negative, and positive predictive values $(96-100 \%){ }^{33,34}$

\section{Evaluation of Stent Patency}

Ultrasonography (US) is the most preferred modality to evaluate the transjugular intrahepatic portosystemic shunt (TIPS), hepatic vein (HV) stent, or SMA stent patency. However, US remains a suboptimal tool in several circumstances, such as obesity and small cirrhotic liver that causes poor beam penetration. Furthermore, artifacts from the air within the fabrics of covered stent graft hinder the evaluation of TIPS patency by US in the early postprocedure period. CT has advantages of wider field of view, three-dimensional reconstruction, and shunt evaluation along with any other addi- tional pathology if there. However, conventional CECT is limited by blooming and beam hardening artifacts from stents that hamper the accurate assessment of in-stent stenosis. Although data regarding the benefits of DECT over SECT in the evaluation of TIPS is lacking, several studies have shown the superiority of DECT over SECT in evaluating peripheral and coronary arterial stent patency. Mangold et al demonstrated reduced blooming artifacts with the improved image quality of peripheral arterial stents at 70 or $80 \mathrm{keV} .{ }^{35}$ Another study by Almutairi et al also showed the superior angiographic image quality of DECT at $72 \mathrm{keV}$ and $50 \%$ ASIR (adaptive statistical iterative reconstruction) in peripheral arterial stenting compared with conventional CTA. ${ }^{36}$ Superior stent lumen visibility for small stents was also reported in a phantom study at high keV when the third generation of DS-DECT and DECT were used. ${ }^{37}$ Owing to superior metallic artifact reduction, DECT could provide superior visibility of TIPS, HV, or SMA stent lumen. In addition, an iodine map could differentiate the patent lumen from in-stent thrombosis (-Fig. 6).

\section{CT-Guided Procedures}

The visibility of lesions during CT-guided biopsy or percutaneous drainage of abdominal collections is significantly reduced by metallic artifacts caused by biopsy/puncture needle that results in inadequate visualization of smaller lesions/vital structures adjoining collections. This increases the chances of false-negative results or inadvertent injury to vital structures. DECT reduces the metal artifacts adequately.

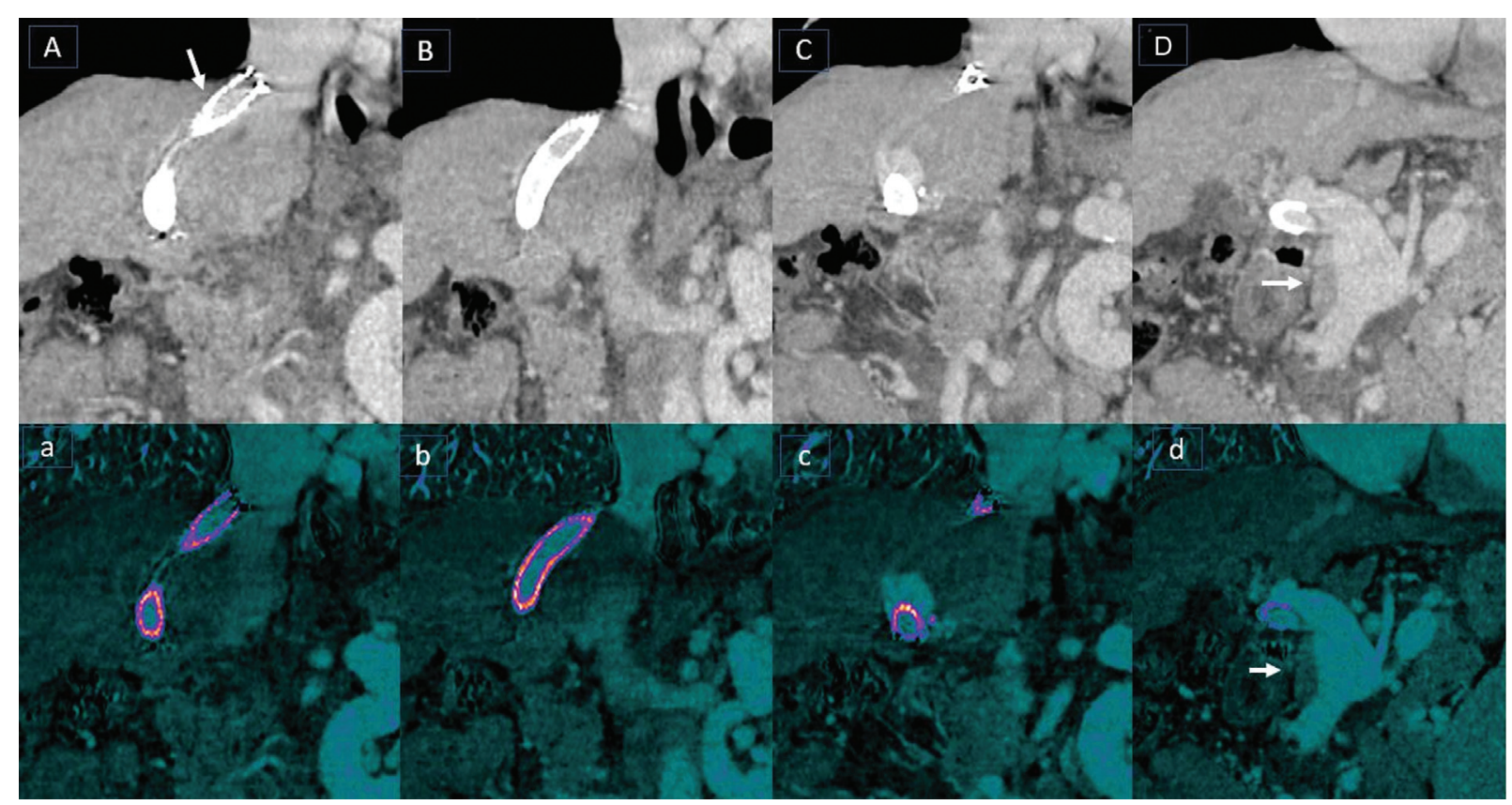

Fig. 6 Dual-energy computed tomography (DECT) scan of abdomen done in a 66-year-old man with transjugular intrahepatic portosystemic shunt (TIPS) which was done for refractory ascites due to chronic liver disease. Contrast-enhanced CT images obtained in the venous phase showing polychromatic images (top row) with corresponding iodine map of monochromatic scan at 65 keV (with a color overlay; bottom row). The iodine maps show better depiction of luminal contrast opacification and reduction of artifacts. (A, a) Hepatic venous end of TIPS stent showing wall-to-wall contrast opacification. (B, b and C, c) Mid stent showing poorly visualized lumen of TIPS stent due to overlapping covered and uncovered stents; however, improved luminal conspicuity on iodine maps was seen. (D, d) Portal venous side of stent. An eccentric, nonocclusive thrombus seen in splenomesenteric confluence. TIPS stent lumen was patent. No intraluminal stent thrombus seen. 


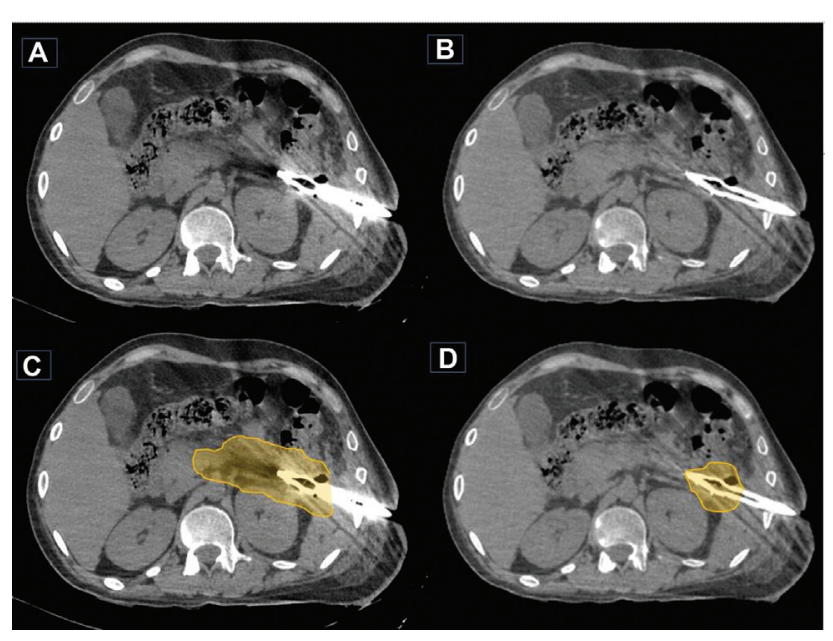

Fig. 7 Imaging using Gemstone spectral imaging metallic artifact reduction software (GSI-MARS) algorithm in a follow-up case of acute necrotizing pancreatitis with drainage catheter in situ. (A, C) Noncontrast computed tomography (NCCT) images with GSI-MARS show lesser artifacts with better visualization of area in the vicinity of catheter tip compared with (B, D) NCCT images obtained without GSIMARS algorithm.

Virtual monochromatic spectral imaging (VMSI) could significantly mitigate metal artifacts during CT-guided biopsy, providing an improved depiction of lesions and safe and versatile access for long puncture pathways. VMSI at $80 \mathrm{keV}$ is usually recommended. Iterative metal artifact reduction can be deployed to mitigate metal artifacts independently of acquisition and reconstruction algorithms ${ }^{38}$ (-Fig. 7).

\section{Limitations of DECT}

Motion and beam hardening artifacts may degrade image quality in most dual-energy current CT systems available currently and must be reduced. ${ }^{38}$ Suboptimal bone removal requires improvement. The major workflow constraints, such as increased reconstruction time, increased volume of images, and increased interpretation time, significantly increase the workload and hinder the use of DECT imaging in routine intervention radiology practice. ${ }^{39}$ To increase the clinical utility of DECT imaging, more versatile dual-energy application algorithms need to be developed.

\section{Conclusion}

In recent years, DECT has been subjected to various advancements, and these can be taken advantage of for better lesion detection and characterization. Different postprocessing techniques not only guide an interventional radiologist in appropriate treatment planning and intraprocedural guidance, but also play an important role in follow-up.

Note

The data sets used and/or analyzed during the current study are available from the corresponding author on reasonable request.

\section{Authors' Contributions}

T.P.T. and K.C. assisted in conceptualizing manuscript; R.P. in images and design of the work; A.M. supported in conceptualizing of the manuscript, manuscript editing, and drafting. All authors read and approved the final manuscript.

\section{Funding}

None.

Conflict of Interest

None declared.

\section{References}

1 Millner MR, McDavid WD, Waggener RG, Dennis MJ, Payne WH, Sank VJ. Extraction of information from CT scans at different energies. Med Phys 1979;6(01):70-71

2 Chiro GD, Brooks RA, Kessler RM, et al. Tissue signatures with dual-energy computed tomography. Radiology 1979;131(02): 521-523

3 Silva AC, Morse BG, Hara AK, Paden RG, Hongo N, Pavlicek W. Dualenergy (spectral) CT: applications in abdominal imaging. Radiographics 2011;31(04):1031-1046, discussion 1047-1050

4 Kalisz K, Halliburton S, Abbara S, et al. Update on cardiovascular applications of multienergy CT. Radiographics 2017;37(07): 1955-1974

5 Khanduri S, Goyal A, Singh B, et al. The utility of dual energy computed tomography in musculoskeletal imaging. J Clin Imaging Sci 2017;7:34

6 Lu GM, Wu SY, Yeh BM, Zhang LJ. Dual-energy computed tomography in pulmonary embolism. Br J Radiol 2010;83(992):707-718

7 Curry TS III, Dowdey JE, Murry RC. Christensen's Physics of Diagnostic Radiology. 4th ed. Philadelphia, PA: Lea \& Febiger; 1990:61-69

8 May MS, Wiesmueller M, Heiss R, et al. Comparison of dual- and single-source dual-energy CT in head and neck imaging. Eur Radiol 2019;29(08):4207-4214

9 Im AL, Lee YH, Bang DH, Yoon KH, Park SH. Dual energy CT in patients with acute abdomen; is it possible for virtual nonenhanced images to replace true non-enhanced images? Emerg Radiol 2013;20(06):475-483

10 Kikano EG, Rajdev M, Salem KZ, et al. Utility of iodine density perfusion maps from dual-energy spectral detector CT in evaluating cardiothoracic conditions: a primer for the radiologist. AJR Am J Roentgenol 2020;214(04):775-785

11 Shuman WP, Green DE, Busey JM, et al. Dual-energy liver CT: effect of monochromatic imaging on lesion detection, conspicuity, and contrast-to-noise ratio of hypervascular lesions on late arterial phase. AJR Am J Roentgenol 2014;203(03):601-606

12 Han SC, Chung YE, Lee YH, Park KK, Kim MJ, Kim KW. Metal artifact reduction software used with abdominopelvic dual-energy CT of patients with metal hip prostheses: assessment of image quality and clinical feasibility. AJR Am J Roentgenol 2014; 203(04):788-795

13 Johnson TR, Krauss B, Sedlmair M, et al. Material differentiation by dual energy CT: initial experience. Eur Radiol 2007;17(06): 1510-1517

14 Purysko AS, Primak AN, Baker ME, et al. Comparison of radiation dose and image quality from single-energy and dual-energy CT examinations in the same patients screened for hepatocellular carcinoma. Clin Radiol 2014;69(12):e538-e544

15 Lehti L, Söderberg M, Höglund P, Nyman U, Gottsäter A, Wassélius J. Reliability of virtual non-contrast computed tomography angiography: comparing it with the real deal. Acta Radiol Open 2018;7 (7-8):2058460118790115 
16 Zhang S, Levin DC, Halpern EJ, Fischman D, Savage M, Walinsky P. Accuracy of MDCT in assessing the degree of stenosis caused by calcified coronary artery plaques. AJR Am J Roentgenol 2008;191 (06):1676-1683

17 Schmid K, McSharry WO, Pameijer CH, Binette JP. Chemical and physicochemical studies on the mineral deposits of the human atherosclerotic aorta. Atherosclerosis 1980;37(02):199-210

18 Uotani K, Watanabe Y, Higashi M, et al. Dual-energy CT head bone and hard plaque removal for quantification of calcified carotid stenosis: utility and comparison with digital subtraction angiography. Eur Radiol 2009;19(08):2060-2065

19 Barrett T, Bowden DJ, Shaida N, et al. Virtual unenhanced second generation dual-source $\mathrm{CT}$ of the liver: is it time to discard the conventional unenhanced phase? Eur J Radiol 2012;81(07): 1438-1445

20 Lv P, Lin XZ, Li J, Li W, Chen K. Differentiation of small hepatic hemangioma from small hepatocellular carcinoma: recently introduced spectral CT method. Radiology 2011;259(03):720-729

21 Wang Q Shi G, Qi X, Fan X, Wang L. Quantitative analysis of the dual-energy CT virtual spectral curve for focal liver lesions characterization. Eur J Radiol 2014;83(10):1759-1764

22 Lee JA, Jeong WK, Kim Y, et al. Dual-energy CT to detect recurrent HCC after TACE: initial experience of color-coded iodine CT imaging. Eur J Radiol 2013;82(04):569-576

23 Brockmann C, Scharf J, Nölte IS, Seiz M, Groden C, Brockmann MA. Dual-energy CT after peri-interventional subarachnoid haemorrhage: a feasibility study. Clin Neuroradiol 2010;20(04):231-235

24 Ferda J, Novák M, Mírka H, et al. The assessment of intracranial bleeding with virtual unenhanced imaging by means of dualenergy CT angiography. Eur Radiol 2009;19(10):2518-2522

25 Gupta R, Phan CM, Leidecker C, et al. Evaluation of dual-energy CT for differentiating intracerebral hemorrhage from iodinated contrast material staining. Radiology 2010;257(01):205-211

26 Hamilton JD, Kumaravel M, Censullo ML, Cohen AM, Kievlan DS, West OC. Multidetector CT evaluation of active extravasation in blunt abdominal and pelvic trauma patients. Radiographics 2008; 28(06):1603-1616

27 Wortman JR, Uyeda JW, Fulwadhva UP, Sodickson AD. Dualenergy CT for abdominal and pelvic trauma. Radiographics 2018;38(02):586-602

28 Golzarian J, Dussaussois L, Abada HT, et al. Helical CT of aorta after endoluminal stent-graft therapy: value of biphasic acquisition. AJR Am J Roentgenol 1998;171(02):329-331
29 Iezzi R, Cotroneo AR, Filippone A, et al. Multidetector CT in abdominal aortic aneurysm treated with endovascular repair: are unenhanced and delayed phase enhanced images effective for endoleak detection? Radiology 2006;241(03):915-921

30 Macari M, Chandarana H, Schmidt B, Lee J, Lamparello P, Babb J. Abdominal aortic aneurysm: can the arterial phase at CT evaluation after endovascular repair be eliminated to reduce radiation dose? Radiology 2006;241(03):908-914

31 Stavropoulos SW, Charagundla SR. Imaging techniques for detection and management of endoleaks after endovascular aortic aneurysm repair. Radiology 2007;243(03):641-655

32 Chandarana H, Godoy MC, Vlahos I, et al. Abdominal aorta: evaluation with dual-source dual-energy multidetector CT after endovascular repair of aneurysms-initial observations. Radiology 2008;249(02):692-700

33 Stolzmann P, Frauenfelder T, Pfammatter T, et al. Endoleaks after endovascular abdominal aortic aneurysm repair: detection with dual-energy dual-source CT. Radiology 2008;249(02):682-691

34 Maturen KE, Kleaveland PA, Kaza RK, et al. Aortic endograft surveillance: use of fast-switch $\mathrm{kVp}$ dual-energy computed tomography with virtual noncontrast imaging. J Comput Assist Tomogr 2011;35(06):742-746

35 Mangold S, De Cecco CN, Schoepf UJ, et al. A noise-optimized virtual monochromatic reconstruction algorithm improves stent visualization and diagnostic accuracy for detection of in-stent restenosis in lower extremity run-off CT angiography. Eur Radiol 2016;26(12):4380-4389

36 Almutairi A, Al Safran Z, AlZaabi SA, Sun Z. Dual energy CT angiography in peripheral arterial stents: optimal scanning protocols with regard to image quality and radiation dose. Quant Imaging Med Surg 2017;7(05):520-531

37 Mangold S, Cannaó PM, Schoepf UJ, et al. Impact of an advanced image-based monoenergetic reconstruction algorithm on coronary stent visualization using third generation dual-source dualenergy CT: a phantom study. Eur Radiol 2016;26(06):1871-1878

38 Do TD, Heim J, Melzig C, et al. Virtual monochromatic spectral imaging versus linearly blended dual-energy and single-energy imaging during CT-guided biopsy needle positioning: optimization of keV settings and impact on image quality. PLoS One 2020; 15(02):e0228578

39 Otrakji A, Digumarthy SR, Lo Gullo R, Flores EJ, Shepard JA, Kalra MK. Dual-energy CT: spectrum of thoracic abnormalities. Radiographics 2016;36(01):38-52 